\title{
Parameter Identification in Population Models for Insects Using Trap Data
}

\author{
Claire Dufourd*, Christopher Weldon ${ }^{\dagger}$, Roumen Anguelov*, Yves Dumont ${ }^{\S}$ \\ * Department of Mathematics and Applied Mathematics, University of Pretoria, Pretoria, South Africa, \\ \{claire.dufourd, roumen.anguelov\}@up.ac.za \\ $\dagger$ Department of Zoology and Entomology, University of Pretoria, Pretoria, South Africa, \\ cwweldon@zoology.up.ac.za \\ $\S$ CIRAD, Umr AMAP, Montpellier, France, \\ yves.dumont@cirad.fr
}

Received: 18 October 2013, accepted: 6 December 2013, published: 23 December 2013

\begin{abstract}
Traps are used commonly to establish the presence and population density of pest insects. Deriving estimates of population density from trap data typically requires knowledge of the properties of the trap (e.g. active area, strength of attraction) as well as some properties of the population (e.g. diffusion rate). These parameters are seldom exactly known, and also tend to vary in time, (e.g. as a result of changing weather conditions, insect physiological condition). We propose using a set of traps in such a configuration that they trap insects at different rates. The properties of the traps and the characteristics of the population, including its density, are simultaneously estimated from the insects captured in these traps. The basic model is an advection-diffusion equation where the traps are represented via a suitable advection term defined by the active area of the traps. The values of the unknown parameters of the model are derived by solving an optimization problem. Numerical simulations demonstrate the accuracy and the robustness of this method of parameter identification.
\end{abstract}

Keywords-partial differential equation; advectiondiffusion equation; parameter identification; inverse problem; trap interference; population density.

\section{INTRODUCTION}

This work is motivated by the need to develop a reliable and efficient method for detecting the presence and estimating population density of the invasive fruit fly, Bactrocera invadens Drew, Tsuruta \& White (Diptera:
Tephritidae) in South Africa. Bactrocera invadens is a fruit fly species introduced from Asia to Africa where it was first described and recorded in Kenya in 2003 [9], [20]. In 2010, B. invadens was detected in the northern part of the Limpopo province in South Africa [21]. Its capacity for rapid population growth, high invasive potential [16], and wide range of fruit hosts [26] represents a major threat for all fruit industries in South Africa.

Fruit flies are a perennial problem in South Africa because in addition to $B$. invadens there are three endemic species that already represent economic pests. Fruit flies have historically been controlled in South Africa by the application of insecticide cover sprays. Current practice, however, involves the use of alternative control strategies due to regulation- and consumer-driven requirements for fruit to be free of insecticide residues. The primary techniques used in fruit fly control are the application of bait sprays [21], M3 bait stations [22], or the 'male annihilation technique' [21]. All three techniques work on the same principal: a food or sex attractant, which is fed on by adult flies, is mixed with an insecticide such as malathion or GF120. With regard to B. invadens, male annihilation technique has been applied to control incursions in South Africa [21]. Another control strategy for this pest may include mass-trapping, which uses male attractants to capture and kill males of a population, leading to reduced female mating and possibly causing local extinction of the population [12]. Alternatively, the 


\section{Dufourd et al., Parameter Identification in Population Models for Insects Using Trap Data}

Sterile Insect Technique (SIT) may represent a useful approach to control incursions of $B$. invadens. SIT involves the release of large numbers of sterilized males that compete with wild males for female fertilization, which leads to no production of viable offspring [18] and its success can be measured using the ratio of sterile: wild insects captured in and array of surveillance traps [17].

Regardless of the alternative control strategy used, their successful application requires a good knowledge of the distribution of the pest, and their dispersal capacity and density. The density of an insect population, however, is a parameter that cannot easily be obtained by direct field observations because traps usually sample only a small proportion of all individuals. To overcome this problem, it is often the case that captures of insects in traps are compared to simulated data [14]. An advection-diffusion equation is considered for modelling the dynamics of fruit flies, where density is the initial value of the model. Such a model requires knowledge of the properties of the trap such as the active area [5] and the strength of attraction, as well as some properties of the population, like its diffusion rate [25]. These parameters are seldom exactly known, and also tend to vary with changing weather [23] and landscape heterogeneity [11],[10].

Determining the values of these parameters is actually an inverse problem, that is, given the solution of the model, or at least part of it, one or more of the model parameters can be identified. The parameter identification problem consists of finding a unique and robust estimation for the parameter values. This problem leads to solving a global optimization problem in order to find the set of parameters that minimizes an objective function. Mathematically, the existence and uniqueness of this global minimum relies on the well-posedness of the inverse problem, while its robustness relies on its well-conditionedness. However, inverse problems are typically ill-posed or conditioned, [19], [7], [24]. In this paper, we show that by using different settings of interfering traps we obtain a parameter identification problem which can be solved numerically in a reliable way. It is essential in this approach that interfering traps generate different incoming streams of insects. Thus, more information about the characteristics of the insect population is provided. Indeed, as the relationship between the setting and the traps is highly non linear and not well understood, several settings of traps are considered and the robustness of the estimates are compared. We demonstrate empirically that using this approach, the problem of simultaneously identifying a set of unknown parameters is well-posed and well-conditioned. The numerical procedure falls under the well-known trial-anderror method of regularization theory [28].

\section{The Insect Trapping Model: The Direct PROBLEM}

The model is formulated on a domain $\Omega \subset \mathbb{R}^{2}$ which is assumed to be isolated, i.e. there is no immigration and no emigration of insects. It is also assumed that when there is no stimulus, the insects individually follow a random walk. Because insects are often in large abundance, we can apply a diffusion equation to model the dispersal of insects at population level [29]. The traps set on $\Omega$ are attractive. Thus, the active area of the trap [5] is the area where the concentration of the attractant is above the threshold of concentration at which the fruit flies can detect it. Therefore, in this area the insects will be influenced to move in a preferred direction towards the trap. This can be modelled using an advection equation [3]. Finally, we assume that our experiments take place over a short period of time, thus we may omit reaction terms.

Using the above assumptions, the insect dynamics can be modelled via an advection-diffusion equation.

$$
\begin{aligned}
& \frac{\partial u}{\partial t}-\nabla(s(x) \nabla u)+\nabla(a(x) u)=0, \\
& \left.\frac{\partial u}{\partial n}\right|_{\partial \Omega}=0, \\
& \left.u\right|_{t=0}=u_{0} .
\end{aligned}
$$

$u(t, x)$ denotes the population density at time $t$ and at the point $x=\left(x_{1}, x_{2}\right) \in \Omega$.

The advection function $a(x)$ is space-dependent and determines the attractiveness of the trap with respect to the distance to the center of the trap. The traps are circular of radius $R_{\text {trap }}$. Assume that the active area of a trap is defined by a disk of radius $R_{\max }$ from the center of the trap. Then the insects that are beyond this disk are not subjected to advection and we assume that their dynamics are only governed by the diffusion term. As the insect gets closer to the trap, the force of attraction increases and reaches its maximum at a distance $R_{\text {min }}$ from the center of the trap. If $N$ is the number of traps distributed on the domain, then:

$$
\begin{aligned}
& a(x)=\sum_{T=1}^{N} a_{T}(x), \\
& a_{T}(x)=a_{\max } \alpha\left(\left\|x_{T}-x\right\|\right) \frac{x_{T}-x}{\left\|x_{T}-x\right\|},
\end{aligned}
$$

where $x_{T}$ is the coordinate of trap $T$, and the function 
$\alpha(d)$ is defined for $d \in[0,+\infty)$, as follows.

$$
\begin{aligned}
& \alpha(d)= \\
& \begin{cases}\frac{a_{\max }}{d} \sin \left(\frac{\pi d}{2 R_{\text {trap }}}\right), & \text { if } d<R_{\text {trap }} \\
\frac{a_{\max }}{d}, & \text { if } R_{\text {trap }} \leq d<R_{\text {min }} \\
\frac{a_{\max }}{2 d}\left(\cos \left(\pi \frac{d-R_{\min }}{R_{\text {max }}-R_{\text {min }}}\right)+1\right) & \text { if } R_{\text {min }} \leq d<R_{\text {max }} \\
0 & \text { if } R_{\text {max }} \leq d\end{cases}
\end{aligned}
$$

The function $\alpha(d)$ is represented in Fig. 11. Note that the value of the advection inside the trap does not really matter, and we make $\alpha(d)$ decrease to 0 from the distance $R_{\text {trap }}$ to ensure the continuity of $a(x)$.

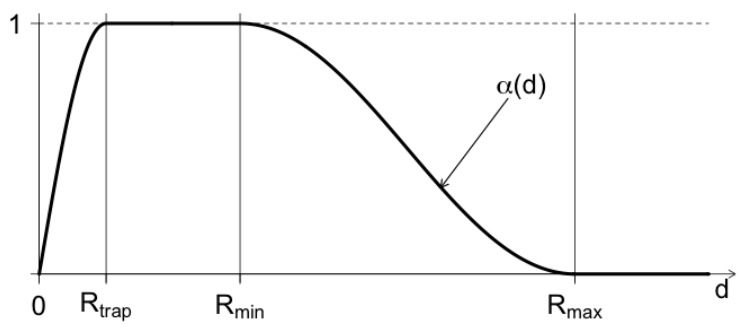

Fig. 1. Graph of the function $\alpha(d)$

The diffusion coefficient $s(x)$ is also space-dependent. It is assumed to be constant, $s(x)=\sigma$, outside the active areas of the traps. Since the insects do not escape from the traps there should be no diffusion across the trap boundary. In order to ensure the existence and uniqueness of the (weak) solution of (1) we assume that inside a trap the function $s(x)$ has a positive value $\varepsilon$ which is so small that the implied diffusion effect in the time interval of observation can be neglected. In order to further ensure continuity of $s$ we take

$$
\begin{aligned}
& s(x)=\sigma-\sum_{T=1}^{N} s_{T}\left(\left\|x_{T}-x\right\|\right), \\
& s_{T}(d)= \\
& \begin{cases}\sigma-\varepsilon, & \text { if } d \leq R_{\text {trap }} \\
(\sigma-\varepsilon)\left(1-\frac{d-R_{\text {trap }}}{R_{\text {min }}-R_{\text {trap }}}\right), & \text { if } R_{\text {trap }}<d \leq R_{\text {min }} \\
0, & \text { if } R_{\text {min }}<d .\end{cases}
\end{aligned}
$$

\section{The Parameter Identification Problem}

The trap parameters, $R_{\min }, R_{\max }$ and $a_{\max }$, as well as the diffusion parameter $\sigma$ are seldom know. Therefore, although our main goal is to estimate the initial population density $u_{0}$, these other parameters are also needed. For simplicity, we assume that the initial population density is a constant, that is, $u_{0}(x)=u_{0} \in \mathbb{R}, \forall x \in \Omega$. Denote $p=\left(u_{0}, \sigma, R_{\min }, R_{\max }, a_{\max }\right)$ the vector of the parameters to identify. Let $\mathcal{P}$ be a compact subset of $\mathbb{R}^{5}$ to which $p$ belongs. When solving the direct problem (1), we are given $p$ and we find a function $u$ satisfying the differential equation in (1) and the respective boundary and initial conditions. This way, we define a mapping $\phi$ from the domain $\mathcal{P}$ of parameters to the space of solutions:

$$
u=\phi(p)
$$

It is well-know that the solution operator $\phi$ is continuous and injective. Therefore, using the compactness of $\mathcal{P}$ we obtain that the operator $\phi^{-1}: \phi(\mathcal{P}) \longrightarrow \mathcal{P}$ is continuous ([28], p.29). Thus, the inverse problem to (1) is wellposed. Then if a solution $u$ of (1) is given, the value of $p=\phi^{-1}(u)$ can be determined by well-known methods, e.g. minimizing a norm of $u-\phi(p)$. However, in practice $u$ is commonly not known, at least not on the whole domain $\Omega \times[0,+\infty)$ [24]. What is usually available is a function $B(u)$ referred to as observation operator [4].

Therefore the parameter identification problem is stated as follows: Given an observation $\psi$, find $p$ such that

$$
(B \circ \phi)(p)=\psi \text {. }
$$

In the setting of problem (1) the observation operator consists of the insect count in traps at given times $t_{1}, t_{2}, \ldots, t_{K}$. More precisely, we have

$$
B(u)=\left(\begin{array}{ccc}
B_{1}\left(u, t_{1}\right) & \ldots & B_{1}\left(u, t_{K}\right) \\
\vdots & & \vdots \\
B_{N}\left(u, t_{1}\right) & \ldots & B_{N}\left(u, t_{K}\right)
\end{array}\right),
$$

where $B_{T}\left(u, t_{k}\right)$ is the total number of insects captured in trap $T$ until time $t_{k}, T=1, \ldots, N, k=1, \ldots, K$. Hence the observation $\psi$ in (6) is an $N \times K$ real matrix

$$
\psi=\left(\begin{array}{ccc}
\psi_{1, t_{1}} & \ldots & \psi_{1, t_{K}} \\
\vdots & & \vdots \\
\psi_{N, t_{1}} & \cdots & \psi_{N, t_{K}}
\end{array}\right)
$$

Note that $B$ is an array of numbers representing averaged values of $u$ on particular areas of the domain at finite number of points in time. Hence, the injectivity of $B \circ \phi$ is problematic. Furthermore, the observation $\psi$ contains both model and measurement error which further complicates the well-posedness of equation (6). In particular, $\psi$ is not necessarily an element of $(B \circ \phi)(\mathcal{P})$. As usual for such situations, $p$ is obtained as a solution of (6) in a least square sense, that is, $p$ is a solution of the optimization problem,

$$
\Phi(p)=\|(B \circ \phi)(p)-\psi\|_{F}^{2} \longrightarrow \min ,
$$


where $\|A\|_{F}=\left(\sum_{i=1}^{m} \sum_{j=1}^{n}\left|a_{i, j}\right|^{2}\right)^{1 / 2}$.

Let $p^{*}$ be the minimizer of $\Phi$, that is

$$
p^{*}=\underset{p \in \mathcal{P}}{\arg \min } \Phi(p)
$$

In the setting of the current model, both the observation operator and the model (1) depend on the distribution of the traps. Our aim is to find trap configurations for which the minimizer of $\Phi$ is unique and can be reliably determined by a numerical procedure. In order to investigate the properties of (7), equivalently (8), we solve the optimization problem iteratively using a random multistart approach [30] over a set of initial values of $p \in \mathcal{P}$. For each randomly selected starting value of $p$, a local minimum of $\Phi$ is found using the Gauss-Newton line search algorithm. The solution of (7) is identified as the estimated parameter values of the minimum of the local minima. Thus, increasing the number of starting values increases the chances of finding the global minimum of the objective function. Furthermore, for each trap setting, we consider how well the global minimum can be discriminated from the other local minima. This gives an important indication on how well the parameter values can be identified in the presence of noise and we refer to it as robustness. Here we investigate the influence of the choice of the settings of traps on the accuracy and robustness of the method.

In general, one can expect that increasing $N$ and $K$ leads to a more regular problem. However, as shown in [2], an incoming stream can be quite accurately identified by using relatively small number of observations. Furthermore, if the traps are far enough from each other, the rows of $B(u)$ are the same. Therefore, in the considered setting, just increasing the size of the matrix $B(u)$ would not improve substantially the regularity of the problem. Our approach is to have some of the traps close enough so that due to interferences, they produce different streams of trapped insects. This will increase the rank of the matrix $B$ which can be reasonably expected to improve the regularity of the problem. Empirical evidence supporting this conjecture is provided in the next section, where the effect of different configuration is examined.

\section{DESCRIPTION OF THE EXPERIMENTS}

We consider four trap settings:

- (A) single trap,

- (B) nine traps in a square formation,
- (C) five traps in a Z-formation,

- (D) five traps in a kite formation.

These four setting are shown on Fig. 2. Note that in all cases the traps are sufficiently far from the boundary of the domain $\Omega$ so that in the considered period of observation the boundary condition in (1) remains reasonable. Fig. 3 represents the population distribution after 15
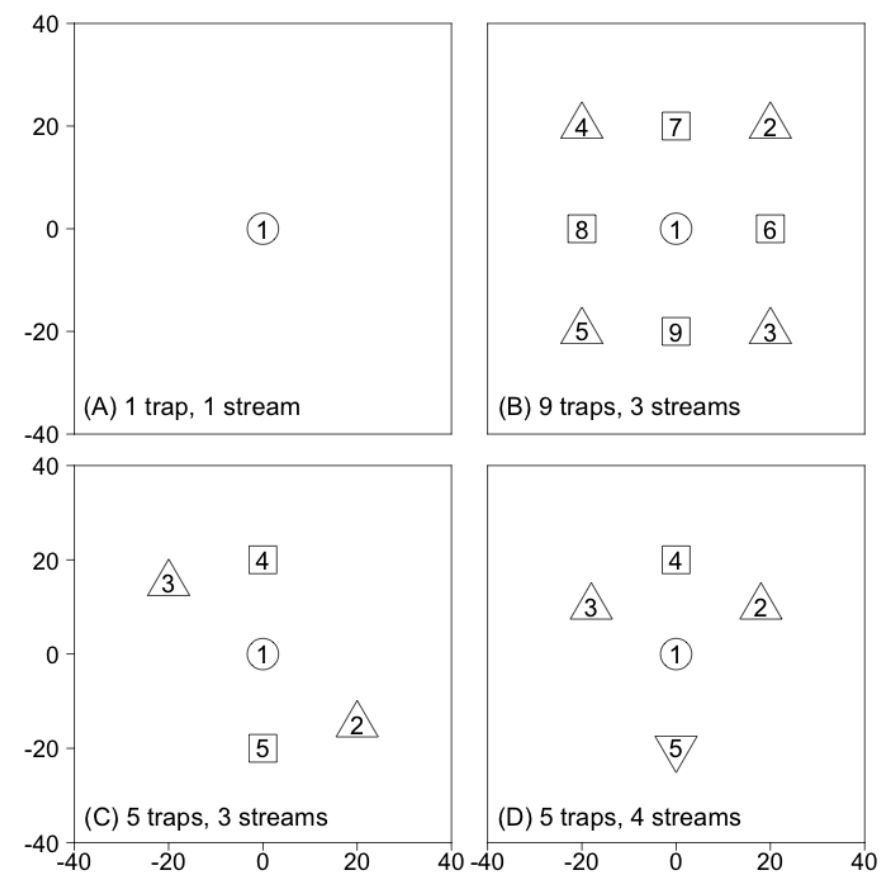

Fig. 2. Distribution of the traps on $\Omega$ for each setting. The symbols identify the traps that produce identical incoming streams within each setting.

time units using setting (D). The interference between the traps can be observed in Fig. 4, which is a zoom in of Fig. 3. Note that in these experiments, because of the symmetry of the trap distribution, identical trap counts are observed in several traps. In Fig. 2, the traps identified with the same symbols have identical incoming streams. Therefore, by using the nine traps of setting (B) for instance, we multiply the amount of information by three compared to the one-trap setting (A). In settings (C) and (D), five traps are used. Due to their configuration, setting (C) produces three distinct incoming streams, whereas setting (D) produces four distinct incoming streams. The distinct incoming streams obtained in each setting are represented in Fig. 5 .

Considering the different settings of traps we run numerical simulations in order to estimate, one, two, three, and finally four parameters simultaneously using the cummulative trap counts over 15 time units $(K=15)$. 


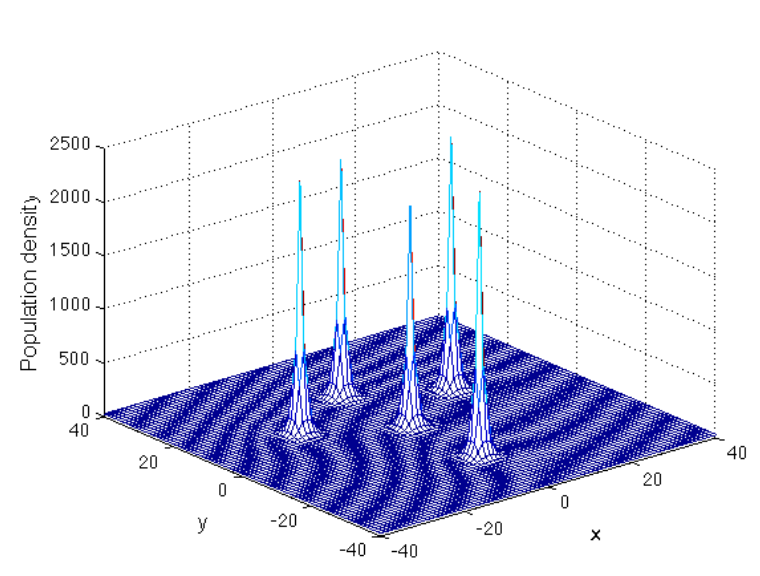

Fig. 3. Insect distribution after 15 time units using setting (D).

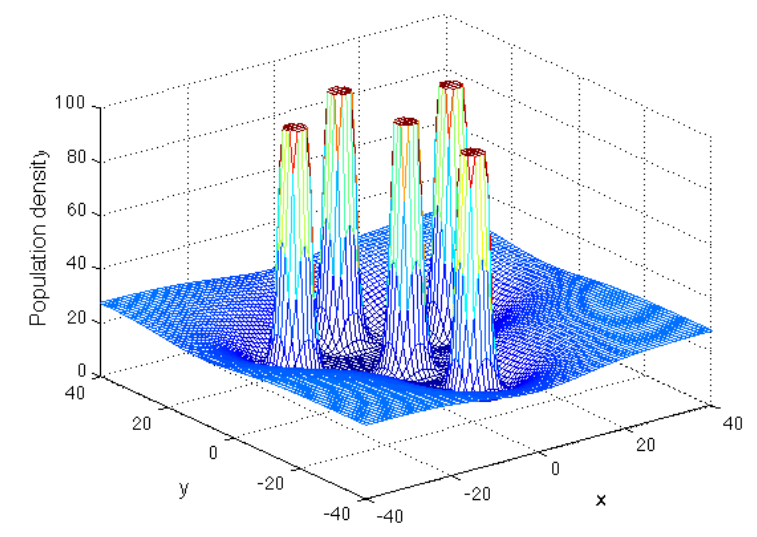

Fig. 4. Interference between the traps using setting (D). Zoom in of Fig. 3

Since the parameter of main interest to identify here is the initial population density $u_{0}$, it is always among the parameters to estimate. The diffusivity of the insects $\sigma$, is the second parameter of main interest when dealing with insect dispersal. The choice of the remaining parameters to estimate is based on a sensitivity analysis. We selected in priority parameters on which the model has the highest sensitivity. Indeed, when solving the inverse problem, the more sensitive the original problem on a parameter is, the more accurate the parameter identification is. The sensitivity of a parameter is a measure of the change in the output of the model caused by a change in this parameter value. However, since the parameters under consideration have different order of magnitude, it is more appropriate to measure their elasticity index [15], i.e. the proportional change in the output of the model caused by a change in the value of the parameter. The

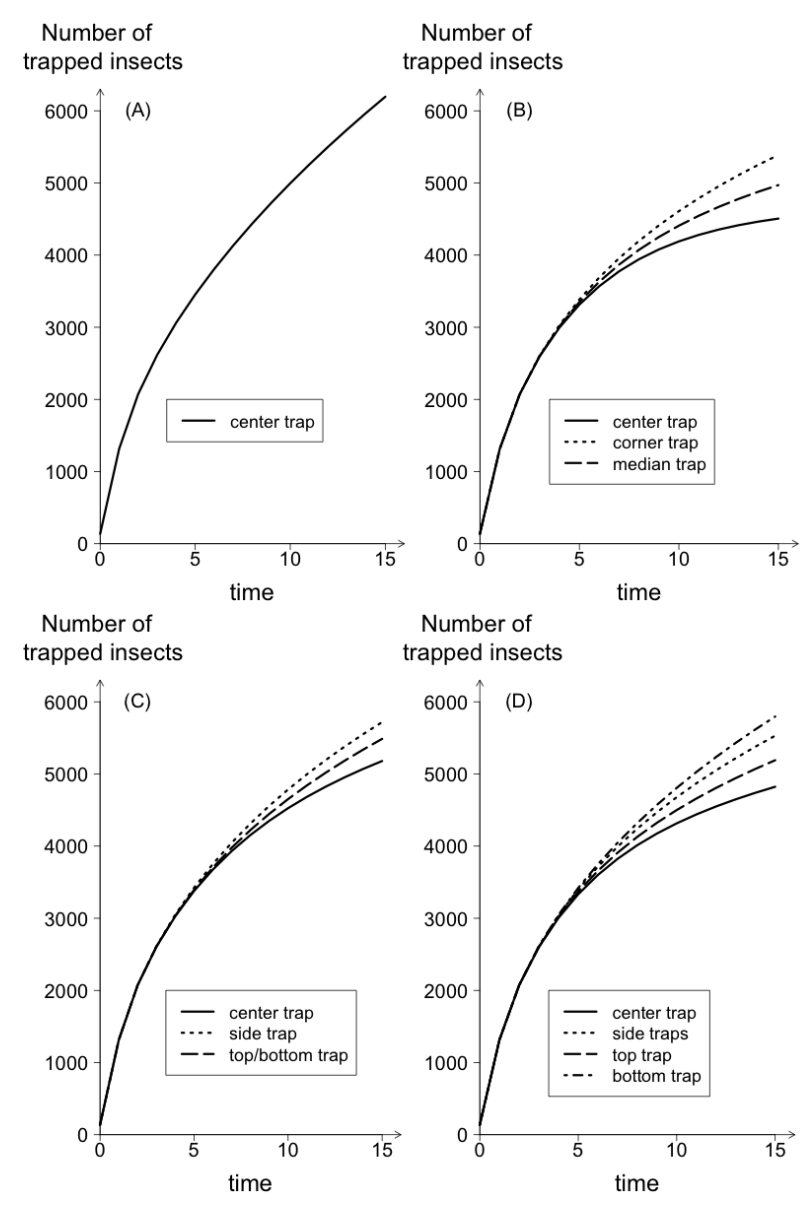

Fig. 5. Cumulative number of captured insects in each trap, using the trap settings (A), (B), (C) and (D) of Fig. 2

elasticity indices of each parameter with respect to each setting of traps is given in Table II. Note that the output of the model is the most sensitive to a change in the parameter for which the elasticity index is the greatest. Therefore, in order the output of the model is more sensitive to changes of values of $a_{\max }$ than of $R_{\max }$ for which the model is more sensitive to changes of $R_{\text {min }}$. Therefore, we proceeded to the parameter identification of $p_{1}=\left(u_{0}\right), p_{2}=\left(u_{0}, \sigma\right), p_{3}=\left(u_{0}, \sigma, a_{\max }\right)$ and $p_{4}=\left(u_{0}, \sigma, a_{\max }, R_{\max }\right)$. Our aim is to find $p_{\kappa}^{*}$ that satisfies (8), where $\kappa$ denotes the number of parameters to be estimated $(\kappa=\{1 ; 2 ; 3 ; 4\})$. We limited our numerical simulations to the simultaneous estimation of 4 parameters due to limitations in computational capacity. Indeed, when solving the inverse problem, each evaluation of the objective function requires solving problem (1).

Since we do not have real field data, we simulated the data over a period of 15 time units with the values of parameters given in Table I. Assuming that the period 


\section{Dufourd et al., Parameter Identification in Population Models for Insects Using Trap Data}

TABLE I

VALUES OF THE PARAMETERS USED TO SIMULATE THE FIELD DATA

\begin{tabular}{llc}
\hline parameter & unit & value \\
\hline$u_{0}$ & insects/unit area & 27.5 \\
$\sigma$ & $\mathrm{m}^{2} /$ time unit & 5.23 \\
$a_{\max }$ & $\mathrm{m} /$ time unit & 5.75 \\
$R_{\max }$ & $\mathrm{m}$ & 8 \\
$R_{\min }$ & $\mathrm{m}$ & 2 \\
\hline
\end{tabular}

TABLE II

ELASTICITY OF THE PARAMETERS WITH RESPECT TO THE INCOMING STREAMS OF INSECTS. LET $\nu$ DENOTE A PARAMETER, AND $z(\nu)$ THE INCOMING STREAM OF INSECTS WITH RESPECT TO $\nu$, THEN, THE ELASTICITY INDEX OF PARAMETER $\nu$ IS $E_{\nu}=\nu\|z(\nu)-z(\nu+\Delta \nu)\|_{2} /\left(\|z(\nu)\|_{2} \Delta \nu\right)$

\begin{tabular}{cl|ccccc}
\hline Setting & Trap & $u_{0}$ & $\sigma$ & $a_{\max }$ & $R_{\max }$ & $R_{\min }$ \\
\hline (A) & & 1.00 & 0.92 & 1.42 & 1.26 & 0.60 \\
\hline \multirow{3}{*}{ (B) } & Center & 1.00 & 1.02 & 1.26 & 0.83 & 0.54 \\
& Corner & 1.00 & 0.96 & 1.35 & 1.06 & 0.57 \\
& Median & 1.00 & 0.98 & 1.31 & 0.95 & 0.55 \\
\hline \multirow{4}{*}{ (C) } & Center & 1.00 & 0.98 & 1.33 & 1.01 & 0.56 \\
& Side & 1.00 & 0.95 & 1.38 & 1.15 & 0.59 \\
& Top/Bottom & 1.00 & 0.95 & 1.36 & 1.08 & 0.58 \\
\hline \multirow{4}{*}{ (D) } & Center & 1.00 & 0.98 & 1.29 & 0.90 & 0.55 \\
& Side & 1.00 & 0.95 & 1.36 & 1.10 & 0.58 \\
& Top & 1.00 & 0.97 & 1.33 & 1.01 & 0.56 \\
& Bottom & 1.00 & 0.94 & 1.39 & 1.17 & 0.59 \\
\hline
\end{tabular}

between two consecutive collects of data is equal to one time unit, we extract from the simulated data only those that correspond the collect time. A noise of $5 \%$ is added to the simulated data, and this gives $\psi=$ $\left(\psi_{i, t_{1}} \ldots \psi_{i, t_{K}}\right)_{i=1 . . N}$, where $N$ is the number of traps. The use of simulated data also allows us to compare the estimation with the real values of the parameters.

The numerical solution of the initial value boundary problem (1) is obtained by using the Crank-Nicolson scheme [8], [27]. The optimisation problem (8) is solved by using the matlab function fminunc which performs the Gauss-Newton line search algorithm [13]. Since this function finds only a local minimum, we run it with many randomly selected initial points in order to have a reasonable certainty that the global minimum is among the local minima found. Here we choose $u_{0}^{(0)} \in[5 ; 45]$, $\sigma^{(0)} \in[4 ; 6], a_{\max }^{(0)} \in[2 ; 8], R_{\max }^{(0)} \in[3 ; 10]$ as ranges of the initial parameter values.

\section{Results AND Discussion}

The results of the experiments described above are presented in Table III. The values of the local minima of $\Phi$, and the respective estimated values of the parameter vectors $\tilde{p}_{\kappa}$, with $\kappa=\{1 ; 2 ; 3 ; 4\}$, are given according to the trap setting. According to our method, when several local minima of $\Phi$ are found, the identified set of parameter values corresponds to the global minimizer of $\Phi$, that is, the minimizer of the smallest local minimum. The $*$ on the right of the table indicates the values of the parameters identified as global minimizers of $\Phi$. In these experiments, we investigate the accuracy and the robustness of the identified parameter values. The accuracy of $p_{\kappa}^{*}$ is measured by calculating the relative error to the exact solution $\bar{p}_{\kappa}$ (Table I) i.e. $E_{r e l}=\left\|\bar{p}_{\kappa}-p_{\kappa}^{*}\right\| /\left\|\bar{p}_{\kappa}\right\|$. In this setting the concept of robustness of the method represents how well the global minimum value of $\Phi$ is discriminated against the values of $\Phi$ of other minima.

TABLE III

LOCAL MIMIMA OF THE OBJECTIVE FUNCTION $\Phi$, WITH THE FOUR SETTINGS OF TRAPS WITH RESPECT TO THE NUMBER OF PARAMETERS ESTIMATED SIMULTANEOUSLY. TS STANDS FOR THE TRAP SETTING USED (FIG. 2). THE* INDICATES THE SET OF PARAMETERS IDENTIFIED AS THE GLOBAL MINIMIZER OF $\Phi$.

\begin{tabular}{|c|c|c|c|c|c|c|c|}
\hline$p$ & $\mathrm{TS}$ & $\Phi$ & $\tilde{u_{0}}$ & $\tilde{\sigma}$ & $\tilde{a}_{\max }$ & $\tilde{R}_{\max }$ & $E_{r e l}$ \\
\hline 2 & (A) & 240004 & 27.63 & - & - & - & $0.5 \% *$ \\
\hline 3 & (B) & 259813 & 27.43 & - & - & - & $0.3 \% *$ \\
\hline$\|$ & (C) & 572150 & 27.45 & - & - & - & $0.2 \% *$ \\
\hline 5 & (D) & 620115 & 27.31 & - & - & - & $0.7 \% *$ \\
\hline \multirow{6}{*}{ 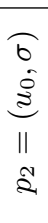 } & (A) & 219149 & 21.49 & 3.31 & - & - & $22.5 \%$ * \\
\hline & & 222543 & 25.43 & 4.73 & - & - & \\
\hline & (B) & 256632 & 27.01 & 5.15 & - & - & $1.8 \% *$ \\
\hline & (C) & 572066 & 27.52 & 5.24 & - & - & $0.1 \% *$ \\
\hline & & 826874 & 19.69 & 2.45 & - & - & \\
\hline & (D) & 619615 & 27.46 & 5.26 & - & - & $0.2 \% *$ \\
\hline \multirow{8}{*}{ 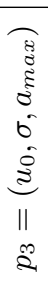 } & (A) & 204279 & 17.79 & 4.30 & 7.89 & - & $34.9 \%$ * \\
\hline & & 214067 & 21.14 & 6.44 & 7.90 & - & \\
\hline & (B) & 256473 & 26.70 & 5.16 & 5.81 & - & $2.8 \% *$ \\
\hline & & 297564 & 18.37 & 4.48 & 7.65 & - & \\
\hline & (C) & 572055 & 27.61 & 5.23 & 5.73 & - & $0.4 \% *$ \\
\hline & & 636640 & 17.35 & 4.18 & 8.07 & - & \\
\hline & (D) & 598689 & 25.17 & 5.48 & 6.31 & - & $8.4 \% *$ \\
\hline & & 647548 & 17.43 & 4.45 & 8.14 & - & \\
\hline \multirow{12}{*}{ 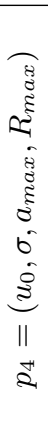 } & (A) & 210462 & 33.31 & 3.25 & 5.07 & 5.83 & $22.1 \%$ * \\
\hline & & 210466 & 34.38 & 3.08 & 4.90 & 5.71 & \\
\hline & & 210471 & 32.75 & 3.35 & 5.17 & 5.89 & \\
\hline & & 210503 & 32.02 & 3.47 & 5.30 & 5.98 & \\
\hline & & 210533 & 31.53 & 3.56 & 5.39 & 6.03 & \\
\hline & & 210679 & 30.22 & 3.81 & 5.65 & 6.20 & \\
\hline & (B) & 255885 & 26.93 & 5.09 & 5.64 & 8.15 & $2.1 \% *$ \\
\hline & & 277462 & 18.24 & 4.00 & 6.80 & 8.56 & \\
\hline & (C) & 571732 & 27.51 & 5.28 & 5.85 & 7.88 & $0.6 \% *$ \\
\hline & & 609828 & 17.47 & 3.84 & 7.05 & 8.61 & \\
\hline & (D) & 597514 & 25.40 & 5.37 & 6.07 & 8.18 & $7.2 \% *$ \\
\hline & & 615604 & 17.66 & 4.06 & 7.08 & 8.61 & \\
\hline
\end{tabular}

As a preliminary experiment, Table IV presents the identified values and relative errors of $p$ when a single parameter value is unknown. $p=\left(u_{0}\right), p=(\sigma), p=$ $\left(a_{\max }\right)$ and $p=\left(R_{\max }\right)$ are successively identified using each setting of traps. These results are in agreement with 


\section{Dufourd et al., Parameter Identification in Population Models for Insects Using Trap Data}

the sensitivity analysis provided in Table II i.e. the more sensitive the output of the model to a certain parameter, the more accurate its estimation.

TABLE IV

IDENTIFIED VALUES OF THE SINGLE VALUE PARAMETER $p$ AND ITS RELATIVE ERROR WITH RESPECT TO THE TRAP SETTING, TS. THE VALUES OF THE ESTIMATES ARE ROUNDED TO TWO DIGITS AFTER THE DECIMAL POINT.

\begin{tabular}{l|l|rrrrrr}
\hline TS & \multicolumn{1}{c|}{$p$} & $\tilde{u}_{0}$ & $\tilde{\sigma}$ & $\tilde{a}_{\max }$ & $\tilde{R}_{\max }$ & $\tilde{R}_{\min }$ & $E_{r e l}$ \\
\hline & $p=\left(u_{0}\right)$ & 27.63 & - & - & - & - & $0.48 \%$ \\
& $p=(\sigma)$ & - & 5.20 & - & - & - & $0.60 \%$ \\
(A) & $p=\left(a_{\max }\right)$ & - & - & 5.77 & - & - & $0.37 \%$ \\
& $p=\left(R_{\max }\right)$ & - & - & - & 8.03 & - & $0.41 \%$ \\
& $p=\left(R_{\min }\right)$ & - & - & - & - & 2.02 & $0.81 \%$ \\
\hline & $p=\left(u_{0}\right)$ & 27.43 & - & - & - & - & $0.24 \%$ \\
& $p=(\sigma)$ & - & 5.24 & - & - & - & $0.21 \%$ \\
& $p=\left(a_{\max }\right)$ & - & - & 5.74 & - & - & $0.17 \%$ \\
& $p=\left(R_{\max }\right)$ & - & - & - & 7.98 & - & $0.24 \%$ \\
& $p=\left(R_{\min }\right)$ & - & - & - & - & 1.99 & $0.64 \%$ \\
\hline & $p=\left(u_{0}\right)$ & 27.45 & - & - & - & - & $0.19 \%$ \\
& $p=(\sigma)$ & - & 5.24 & - & - & - & $0.19 \%$ \\
& $p=\left(a_{\max }\right)$ & - & - & 5.74 & - & - & $0.13 \%$ \\
& $p=\left(R_{\max }\right)$ & - & - & - & 7.99 & - & $0.17 \%$ \\
& $p=\left(R_{\max }\right)$ & - & - & - & - & 1.99 & $0.05 \%$ \\
\hline & $p=\left(u_{0}\right)$ & 27.31 & - & - & - & - & $0.69 \%$ \\
& $p=(\sigma)$ & - & 5.27 & - & - & - & $0.71 \%$ \\
& $p=\left(a_{\max }\right)$ & - & - & 5.72 & - & - & $0.49 \%$ \\
& $p=\left(R_{\max }\right)$ & - & - & - & 7.95 & - & $0.60 \%$ \\
& $p=\left(R_{\min }\right)$ & - & - & - & - & 1.96 & $1.76 \%$ \\
\hline & & & & & & &
\end{tabular}

\section{A. Do interfering trap-settings provide better results} than non-interfering trap settings?

From Table III we can see that, using setting (A) i.e. without trap interference, the number local minima found increases, as the number of parameters to identify simultaneously increases. Conversely, using the setting with trap interferences, a maximum of two minima were found.

Moreover, the minima found using one trap are in a maximum range of $5 \%$ of the optimal value of $\phi$ and within this range, the norms of the different minima can differ by $15 \%$. More precisely, for $p=p_{2}$ (resp. $\left.p=p_{3}\right)$, the minima are found withing a range of $2 \%$ (resp. $15 \%$ ) of the optimal value of $\phi$. In particular, when $p=p_{4}, 6$ minima are found within a range of $0.1 \%$ of the optimal value of $\phi$ where the norm of the minima can differ by $10.6 \%$. This shows that, without interferences, as the number of parameters to identify increases, the reliability of the estimates decreases.

Furthermore, we can see from Table III], that the one-trap setting provides a poor accuracy of the estimates when several parameter values are identified simultaneously, compared to the other setting. In fact, using one trap, the relative error of the estimates is above $22 \%$ when two or more parameter values are estimated, whereas, using the other settings, the relative error is always below $8.4 \%$. Therefore, when two or more parameters are to be identified, we may conclude that interfering trap settings provide estimates with better accuracy than non-interfering trap settings. However, from Table III], note that if a single parameter needs to be identified, setting (A), using only one trap, would provide a sufficiently accurate and reliable estimate. In this case, adding more traps is not really helpful. This suggests that one must choose an appropiate setting depending on the parameters that need to be identified.

By investigating further on the results, when $p=p_{3}$ using one trap, we simulated the incoming streams using the global minimizer of $\Phi\left(p_{3}=p_{3}^{*}\right)$, (Table III). The curve obtained with the latter simulation as well as the curve representing the incoming stream simulated using the real value of $p$, given in Table $\mathbb{\Pi}\left(p_{3}=\bar{p}_{3}\right)$, are represented in Fig. 6. As we can see, the two curves are hardly distinguishable, meaning that two different sets of parameters can lead to very similar incoming streams. This illustrates the ill-conditionedness of the problem where two very similar streams of trapped insects are produced using two very different sets of parameters $\left(E_{r e l}=34.9 \%\right.$, Table IIII).

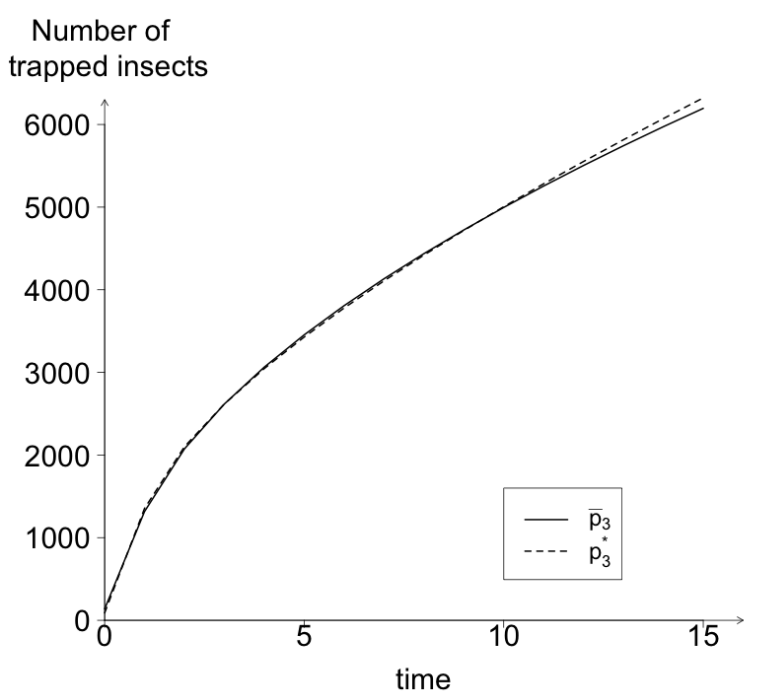

Fig. 6. Simulated cumulative number of captures when estimating three parameters with setting (A), using the real values, $\bar{p}_{3}$, and the identified values, $p_{3}^{*}$.

\section{B. Interfering trap-setting strategies}

We have shown above that using a setting of traps that are interfering provide better results for parameter identi- 


\section{Dufourd et al., Parameter Identification in Population Models for Insects Using Trap Data}

fication in terms of robustness and accuracy when several parameters are identified simultaneously. However, little is known on the actual role of the interferences between the traps and their effect on the regularity of the problem. In order to understand the effect of these interactions, several interfering trap settings are compared.

We study the results obtained using the nine-trap setting (B) and the setting (D) of five traps. One could expect better accuracy and robustness of the estimates using setting (D) since in this setting four distinct incoming streams are produced whereas only three are produced using setting (B). However, apart from the case $p=p_{1}$, the relative error of the estimate using setting (D) is always lower than using setting (B). Considering the fact that the noisy data are averaged over the traps producing the same streams, in setting (B), the corner and median noisy data are averaged over three traps, whereas only the noisy data of the two side traps are averaged using setting (D). Therefore, the data used to identify the parameters using setting (B) are smoother than those used with setting (D). This reduces the effect of the noise and may explain why more accurate and robust estimations are obtained using nine traps.

In setting (C), five traps are used producing three distinct incoming streams as for the nine-trap setting (B). The estimates obtained using setting (C) are the most accurate compared to all the other experiments that were carried out $\left(E_{r e l}=6 \%\right.$ for $\left.p=p_{4}\right)$. The robustness, however, is not as good as for the nine trap setting. For instance, when $p=p_{3}$, two local minima of $\phi$ are found within $10 \%$ (resp. 14\%) of its optimal value for setting (C) (resp. setting (B)). Despite this, the global minimum of $\phi$ is clearly identified. This counter intuitive result shows that the interference phenomenon is not trivial. In particular, we can see in Fig. 5 that the 3 incoming streams produced by the nine trap setting are more distinct than those obtained using the five-trap setting. A possible explanation may relate to the sensitivity of the parameters. Indeed, the parameters $a_{\max }$ and $R_{\max }$ are more sensitive using setting (C) than when using setting (B) (Table III). Such a result suggest that there must be an optimal setting which would allow to obtain robust and accurate parameter estimation using as few traps as possible.

These results show that trap interference can be used to make the problem more regular, however, the relationship between the regularity of the problem and the interfence between the traps is highly nonlinear and therefore difficult to analyse.

\section{CONCLUSION}

Parameter Identification is challenging, in particular when the direct problem is defined by PDEs, since it often leads to solving inverse problems that are illposed or ill-conditioned. We demonstrate numerically that the interferences between traps can be used to make the problem well-posed and well-conditioned using a trial-and-error approach. This method enables the identification of parameter values that describe population characteristics, i.e the population density, its diffusion rate, attractiveness of the traps and their maximum radius of attraction. By choosing biologically realistic ranges of parameter values to estimate, the parameters are identified as the global minimizer of function $\Phi$ using starting values over these ranges. We show numerically that trap interferences can be used not only to increase the accuracy of the estimates, but also to find a global minimum which can be well descriminated from the other local minima. The trial-and-error method over the choice of the trap setting, followed with the multistart approach to solve the optimization method increases the chances of succeeding in solving the parameter identification problem. For each trap setting trial, we found numerically the global minimum of the objective function and we measured how well it is discriminated from the other minima. The setting where the global minimum can be the best discriminated from the other minima provides an inverse problem that is well-conditioned and thus provides the most robust solution to the parameter identification problem. Note that, once a "good" setting of trap is identified, other methods, such as random search methods, could be interesting alternatives to finding the global minimum of the objective function [30], [6], [1].

Furthermore this work investigates how experiments using traps should be conducted in the field so that sufficient information is recorded, using as few traps as possible. Indeed, the optimal setting of traps depends on the parameters that need to be identified. For instance, we showed that a setting using only one trap would be sufficient to identify a single parameter. However when several parameters are unknown, interfering traps provide more accurate and more reliable estimates. In particular, setting (C) stands out from the other settings. Due to a highly nonlinear relationship between the trap interferences and the regularity of the problem, it is challenging to find an optimal setting of traps providing highly accurate and robust estimates. A global sensitivity of the model to the parameters will be considered in a future work to give more insight into this relationship. 


\section{Dufourd et al., Parameter Identification in Population Models for Insects Using Trap Data}

This is a promising method, not only from the numerical and theoretical perspective for parameter identification, but may also prove to be of practical importance for the determination of insect population density in the field with the use of traps.

\section{ACKNOWLEGMENT}

The support of the National Research Fundation of South Africa and Citrus Research International Ltd. (Project: 1075) is acknowledged. Y. Dumont would also like to thank the support of the "SIT feasibility program" in Reunion, jointly funded by the French Ministry of Health and the European Regional Development Fund (ERDF).

\section{REFERENCES}

[1] V.V. Akimenko, Yu. V. Zahorodnii, A.L. Boyko, "Identification of parameters of evolutionary model of monocyclic cels aggregation with the hop plants example" Computers \& Mathematics with Applications, vol. 66, pp. 1547-1553, 2013. http://dx.doi.org/10.1016/j.camwa.2013.02.023

[2] H.T. Banks and P. Karreiva, "Parameter estimation techniques for transport equations with application to population dispersal and tissue bulk flow models." Journal of Mathematical Biology, vol. 17 , no. 3 , pp. $253-273,1983$. http://dx.doi.org/10.1007/BF00276516

[3] H.T. Banks; P. Karreiva and L. Zia, "Analyzing field studies of insect dispersal using two-dimensional transport equations." Environmental entomology, vol. 17, no. 5, pp. 815-820, 1988.

[4] M. Burger, "Parameter Identification," Lecture Notes, 2005.

[5] J.A. Byers, "Active space of pheromone plume and its relationship to effective attraction radius in applied models." Journal of chemical ecology, vol. 34, no. 9, pp. 1134-1145, $20 \overline{08 .}$ http://dx.doi.org/10.1007/s10886-008-9509-0

[6] I-C. Chou, E.O. Voit, "Recent developments in the parameter estimation and structure identification of biochemical and genomic systems." Mathematical Biosciences, vol. 219, no. 2, pp. 57-83, 2009. http://dx.doi.org/10.1016/j.mbs.2009.03.002

[7] M. Cristofol; and J. Garnier; and F. Hamel; and L. Roques, "Uniqueness from pointwise observations in a multi-parameter inverse problem" Communication on Pure and Applied Analysis, vol. 11, no. 1, pp. 173-188, 2012. http://dx.doi.org/10.3934/cpaa.2012.11.173

[8] J. Crank and P. Nicolson, "A practical method for numerical evaluation of solutions of partial different" Mathematical Proceedings of the Cambridge Philosophical Society, vol. 43, no. 1, pp. 50-67, 1947. http://dx.doi.org/10.1007/BF02127704

[9] R.A.I. Drew, K. Tsuruta and I.M. White, "A new species of pest fruit fly (Diptera: Tephritidae: Dacinae) from Sri Lanka and Africa." African Entomology, vol. 13, no. 1, pp. 149-154, 2005.

[10] C. Dufourd and Y. Dumont, "Modeling and Simulations of mosquito dispersal. The case of Aedes albopictus," Biomath, vol. 1, no. 2, pp. 128-134, 2012. http://dx.doi.org/10.11145/j.biomath.2012.09.262
[11] C. Dufourd and Y. Dumont, "Impact of environmental factors on mosquito dispersal in the prospect of sterile insect technique control," Computers and Mathematics with Applications, vol 66, pp. $1695-1715,2013$. http://dx.doi.org/10.1016/j.camwa.2013.03.024

[12] A.M. El-Sayed, D.M. Suckling, C.H. Wearing and J.A. Byers "Potential of mass trapping for long-term pest management and eradication of invasive species," Journal of economic entomology, vol. 99, no. 5, pp. 1550-1564, 2006. http://dx.doi.org/10.1603/0022-0493-99.5.1550

[13] R. Fletcher, "Practical methods of optimization.", vol. 1, Unconstrained Optimization, John Wiley and Sons, 1980.

[14] A. Hartstack, J. Witz. "Estimating field populations of tobacco budworm moths from pheromone trap catches.," Environmental Entomology, vol. 10, no. 6, pp. 908-914, 1981.

[15] J.R. Hicks, TValue ans capital. Oxford University Press, Oxford, England, 1976.

[16] F.M. Khamis, N. Karam, S. Ekesi, M. De Meyer, A. Bonomi, L.M. Gomulski, F. Scolari, P. Gabrieli, P. Siciliano, D. Masiga, E.U. Kenya, G. Gasperi, A.R. Malacrida, C.R. Guglielmino, "Uncovering the tracks of a recent and rapid invasion: the case of the fruit fly pest Bactrocera invadens (Diptera: Tephritidae) in Africa.", Molecular Ecology, vol. 18, no. 23, pp. 4798-4810, 2009. http://dx.doi.org/10.1111/j.1365-294X.2009.04391.x

[17] E.F. Knipling, "Possibilities of insect control or eradication through the use of sexually sterile males.", Journal of Economic Entomology, vol. 48, no. 4, pp. 459-462, 1955.

[18] D.R. Lance and D.O. Mclnnis, "Biological basis of the sterile insect technique.” In V.A. Dyck, J. Hendrich and J.S. Robinson, Sterile insect technique: principles and practice in area-wide integrated pest management, Springer, pp. 69-94, 2005. http://dx.doi.org/10.1007/1-4020-4051-2_3

[19] M.M. Lavrentev, V.G. Romanov, S.P. Shishatskii, Ill-posed problems of mathematical physics and analysis, AMS, vol. 64, 1986.

[20] S.A. Lux, R.S. Copeland, I.M. White and A. Manrakhan, "A new invasive fruit fly species from the Bactrocera dorsalis (Hendel) group detected in East Africa." Insect Science and its Application, vol. 23, no. 4 , pp. 355-361, 2003. http://dx.doi.org/10.1017/S174275840001242x

[21] A. Manrakhan, V. Hattingh, J.H. Vente and M. Holtzhausen, "Eradication of Bactrocera invadens (Diptera: Tephritidae) in Limpopo Province, South Africa.” African Entomology, vol. 19, no. 3 , pp. 650-659, 2011. http://dx.doi.org/10.4001/003.019.0307

[22] A. Manrakhan, C. Kotze, "Attraction of Ceratitis capitata, C. rosa and C. cosyra (Diptera: Tephritidae) to proteinaceous baits." Journal of Applied Entomology, vol. 135, pp. 98-105, 2011. http://dx.doi.org/10.1111/j.1439-0418.2009.01479.x

[23] M.W. Mwatawala, M. De Meyer, R.H. Makundi and A.P. Maerere, "Seasonality and host utilization of the invasive fruit fly, Bactrocera invadens (Dipt., Tephritidae) in central Tanzania." Journal of Applied Entomology, vol. 130, no. 9-10, pp. $530-537,2006$. http://dx.doi.org/10.1111/j.1439-0418.2006.01099.x

[24] A. Perasso, Identifiabilité de paramètres pour des systèmes dcrits par des quations aux dérivées partielles. Application la dynamique des populations. Ph.D. Thesis, Universit Paris SudParis XI: France, 2009. http://tel.archives-ouvertes.fr/tel-00464272

[25] S. Petrovskii, D. Bearup, D.A. hmed ,R. Blackshaw, "Esti- 
C Dufourd et al., Parameter Identification in Population Models for Insects Using Trap Data

mating insect population density from trap counts." Ecological Complexity, vol. 10, pp. 69-82, 2012.

http://dx.doi.org/10.1016/j.ecocom.2011.10.002

[26] I. Rwomushana, S. Ekesi, I. Gordon, C.K.P.O. Ogol "Host plants and host plant preference studies for Bactrocera invadens (Diptera: Tephritidae) in Kenya, a new invasive fruit fly species in Africa." Annals of the Entomological Society of America, vol. 101, pp. 331-340, 2008. http://dx.doi.org/10.1603/0013-8746(2008)101[331: HPAHPP]2.0.CO;2

[27] J. Strikwerda, Finite difference schemes and partial differential equations. Siam, 2007. http://dx.doi.org/10.1137/1.9780898717938

[28] A.N. Tikhonov, V.Y. Arsenin, Solution of Ill-posed Problems. Washington: Winston \& Sons, 1977.

[29] P. Turchin, Quantitative analysis of movement: measuring and modeling population redistribution in animals and plants. Sinauer Associates Sunderland, 1998.

[30] A.A. Zhigljavsky, Theory of Global Random Search. Kluwer Academic Publishers, Dordrech, 1991. 